\title{
GLP-1R Activation Ameliorate Novel-object Recognition Function via Hippocampal AMPK/NF- KB Signaling Pathway in Neuropathic Pain Mice
}

\section{Long-Qing Zhang}

Tongji Hospital of Tongji Medical College of Huazhong University of Science and Technology

\section{Wen Zhang}

Tongji Hospital of Tongji Medical College of Huazhong University of Science and Technology

\section{Ting Li}

Tongji Hospital of Tongji Medical College of Huazhong University of Science and Technology

\section{Ting Yang}

Tongji Hospital of Tongji Medical College of Huazhong University of Science and Technology

\section{Xiaoman Yuan}

Tongji Hospital of Tongji Medical College of Huazhong University of Science and Technology

\section{Yaqun Zhou}

Tongji Hospital of Tongji Medical College of Huazhong University of Science and Technology

\section{Qian Zou}

Tongji Hospital of Tongji Medical College of Huazhong University of Science and Technology

\section{Hui Yang}

Tongji Hospital of Tongji Medical College of Huazhong University of Science and Technology

\section{Feng Gao}

Tongji Hospital of Tongji Medical College of Huazhong University of Science and Technology Yuke Tian

Tongji Hospital of Tongji Medical College of Huazhong University of Science and Technology

\section{Wei Mei}

Tongji Hospital of Tongji Medical College of Huazhong University of Science and Technology Xue-Bi Tian ( $\nabla$ tianxb@hust.edu.cn )

Tongji Hospital of Tongji Medical College of Huazhong University of Science and Technology https://orcid.org/0000-0002-0624-6816

\section{Research}

Keywords: GLP-1R, neuropathic pain, memory, neuroprotection, neuroinflammation

Posted Date: October 27th, 2020 
DOI: https://doi.org/10.21203/rs.3.rs-96271/v1

License: (c) (1) This work is licensed under a Creative Commons Attribution 4.0 International License. Read Full License 


\section{Abstract}

Background: Growing evidences indicate that neuropathic pain is frequently accompanied with cognitive impairments, which aggravate the decrease in the quality of life of chronic pain patients. Furthermore, it has been shown that the activation of Glucagon-like-peptide-1 receptor (GLP-1R) improved memory deficit in multiple diseases such as Alzheimer's disease (AD), stroke and so on. However, whether activating GLP-1R could improve memory impairment induced by neuropathic pain and the mechanism by which this improvement would occur remain unclear.

Methods: The spared nerve injury (SNI) model was established as a kind of neuropathic pain. And novelobject recognition memory (hippocampus-dependent memory) was tested by the novel object recognition test (NORT). The expression levels of GLP-1,GLP-1R,adenosine monophosphate-activated protein kinase (AMPK),p-AMPKThr172, nuclear factor k B p65 (NF-kB p65), interleukin-1 $\beta$ p17(IL-1 $\beta$ p17), and the synaptic associated proteins were tested in the murine hippocampus with memory deficits caused by neuropathic pain. Then, exenatide acetate (Ex-4, a GLP-1R agonist), exendin(9-39)(Ex(9-39),a GLP-1R antagonist) and Compound $C$ dihydrochloride ( $C C$, an AMPK inhibitor) were used to test the effects of activating GLP-1R in mice with neuropathic pain.

Results: First, we uncovered that neuropathic pain could inhibit GLP-1/GLP-R axis, disturb inflammatory signaling pathway, increase the expression of IL-1 $\beta$ and downregulate the synaptic associated proteins (postsynaptic density protein 95(PSD95) and Arc). Subsequently, we reported that Ex-4 treatment could improve recognition memory impairment and increase the ratio of p-AMPKThr172/AMPK, decrease the expression of NF-KB p65 and IL-1 $\beta$ p17, upregulate the levels of PSD95 and Arc. Moreover, we uncovered that $\operatorname{Ex}(9-39)$ and $C C$ treatment could abrogate the neuroprotection of Ex-4 in mice with memory impairment induced by neuropathic pain.

Conclusions: The results indicated that the activation of GLP-1R could improve recognition memory impairment caused by neuropathic pain via activating AMPK signaling pathway, then, which could inhibit NF-KB activating and its downstream IL-1 $\beta$ expression, upregulate the levels of PSD95 and Arc proteins.

\section{Backgroud}

There are a large number of patients with chronic pain worldwide, and approximately $20 \%$ of patients suffer from cognitive deficit [1]. Although, several studies reported that chronic pain can induce cognitive impairment [2-4], the mechanism of memory dysfunction caused by chronic pain is exceedingly complex, leading to lack of effective prevention and treatment measures.

Glucagon-like-peptide-1receptor belongs to the G protein coupled receptors (GPCRs) family, which is expressed broadly throughout the brain, including the hippocampus [5]. Some studies have shown that activating GLP-1R can promote long-term potentiation (LTP) and that GLP-1R participates in learning and memory [6].Additionally, activating GLP-1R also can mitigate neuroinflammation [7, 8]. Furthermore, 
neuroinflammaton is a critical factor contributing to hippocampus-dependent memory impairment [9].Neuropathic pain can alos cause the release of pro-inflammatory cytokines in the hippocampus [3].

It has been reported that AMPK, as a downstream of GLP-1R, can be activated when activating GLP-1R [10-12].Furthermore, the activation of AMPK can inhibit the downstream protein p65 NF-KB, which is involved in controlling neuroinflammation $[13,14]$. Additionally, activation of the NF-KB could promote the secretion of multiple pro-inflammatory cytokines including IL-1 $[14,15]$, which contribute to neuropathic pain-induced memory impairment $[16,17]$, and IL-1 $\beta$ p 17 is a mature fragment of IL-1 $1 \beta$.In addition, the activation of GLP-1R can attenuate the increased levels of IL-1 $\beta$ in the brain $[8,18]$.

However, little is known about the neuroprotection of GLP-1R activation during the chronic pain phase. Also, whether the activation of GLP-1R could improve memory impairment induced by SNI, and the specific mechanism by which this improvement would occur are still unclear. Here, we hypothesized that activating the GLP-1R/AMPK signaling pathway would attenuate memory deficits induced by neuropathic pain via the inhibition of neuroinflammation, raising the levels of synaptic associated proteins.

\section{Methods}

\section{Animals}

Male C57 BL/6(7-8 weeks) mice were purchased from Tongji Hospital, Tongji Medical College, Huazhong University of Science and Technology, Wuhan, Hubei, China. Mice were raised under controlled conditions $\left(22-25^{\circ} \mathrm{C}, 12\right.$-hour alternate circadian rhythm, food, with food and water ad libitum). All animal studies were approved by the Animal Care and Use Committee of Tongji hospital.

\section{Induction of neuropathic pain}

The establishment of SNI models was according to Decosterd and Woolf[19].Briefly, under pentobarbital sodium anesthesia ( $50 \mathrm{mg} / \mathrm{kg}$, intraperitoneally), the left sciatic nerve (including the 3 branches: common peroneal , tibial, sural nerve) was exposured. The 6.0 silk thread (or suture) was tightly ligated with the two branches of the sciatic nerve (common peroneal nerve and tibial nerve). The distal end of the ligation site was cut off, and the distal nerve stump of 2-4 mm was removed. For the sham group, we only exposed the sciatic nerve without ligation.

\section{Pain behavior test}

The ipsilateral hind paw mechanical paw withdrawal threshold (MPWT) was measured using Von Frey filament $(0.02,0.04,0.07,0.16,0.4,0.6$, and $1 \mathrm{~g})$, which represented mechanical allodynia as previously described [20] (Behavioral tests were conducted between 8:30 AM and 16:30 PM).

\section{Novel object recognition test}


The NORT was conducted as previously described [9]. Briefly, the mice were handled for 1 minute a day continuing 6 days before the test. Then, mice were put into the box and adapted to the condition for 5 minutes. In the training section, the mice were allowed to explore two identical rectangular blocks (familiar objects) for 5 minutes and rectangular blocks were placed on the same side of the box. Sniffing, licking, and climbing on objects were all regarded as the exploratory behaviors. In the testing phase, a rectangular block was superseded by a cylinder (novel object), and mice were allowed to explore the two objects for another 5 minutes in the box. The cognitive function was evaluated by the discrimination ratio (DR) which is represented by $T_{C} /\left(T_{R}+T_{C}\right)$, where $T_{C}$ is the time spent exploring the novel object, $T_{R}$ is the time spent exploring the familiar object, and $T_{R}+T_{C}$ is the total time spent exploring the two objects.

\section{West Blot analysis}

Collection of hippocampal tissue and preparation of hippocampal protein samples were according before [1].Briefly, equal amounts of hippocampal proteins samples were separated using $10 \%$ sodium dodecyl sulfatepolyacrylamide gel electrophoresis (SDS-PAGE) and subsequently transferred to polyvinylidene fluoride membranes (Millipore) for electroblotting. The membranes were blocked with $5 \%$ bovine serum albumin (BSA) in Tris-buffered saline and Tween 20 (TBST, 0.1\%) for 2 hours at routine temperature (RT), incubated with primary antibody overnight at $4{ }^{\circ} \mathrm{C}$, and then incubated with horseradish peroxidase (HRP)conjugated goat anti-mouse secondary antibody (1:5000;ABclonal) or goat anti-rabbit secondary antibody(1:5000;Aspen) for 2 hours at RT. The specific primary antibodies used in this study include mouse anti-glyceraldehyde 3-phosphate dehydrogenase (GAPDH) (1:2000;ABclonal); rabbit anti-GLP1(1:500;ABclonal);rabbit anti-GLP-1R (1:1000;ABclonal); rabbit anti-PSD95 (1:1000; Cell Signaling Technology); rabbit anti-Arc (1:1000;ABclonal); rabbit anti-AMPK (1:1000; Cell Signaling Technology); rabbit anti-p-AMPKThr172 (1:1000; Cell Signaling Technology); rabbit anti-NF-KBp65 (1:1000; Proteintech), rabbit anti-pNF-kBp65 (1:1000; Cell Signaling Technology); rabbit anti-IL-1ß p17 (1:1000; Affinity). The protein bands were tested using chemiluminescence (Pierce ECL Western Blotting Substrate; Thermo Scientific) and measured using a computerized image analysis system (ChemiDoc XRS+; BIORAD).

\section{Experimental designs and Drugs treatment}

There were four experiments in this study, as shown in (Fig 1A, 3A, 5A, 7A). First, we study whether neuropathic pain could induce recognition memory impairment. Then, to examine whether activating GLP-1R could alleviate memory impairment caused by neuropathic pain, Ex-4 $(0.4 \mathrm{mg} / \mathrm{kg}$, purchased from Selleck.cn) or Vehicle, was subcutaneously (s.c.) administrated into mice, starting from day 1 to day 21 after surgery. The dosage of Ex-4 was selected according to previous research [21]. Ex(9-39) $(0.1 \mathrm{mg} / \mathrm{kg}$, purchased from MedChemExpress) was intraperitoneally (i.p.) administered into mice 30 min before Ex-4 treatment from day 1 to day 21 after operation. The selection of the dosage and administration route for Ex(9-39) was based on previous research [22].Finally, to test the neuroprotective mechanism of activating GLP-1R, CC(20mg/kg, purchased from MedChemExpress) was given to mice via intraperitoneal injection 
30 min before Ex-4, from day 1 to 21 after nerve injury. The dosage of CC was selected depending on previous research [23].

\section{Statistical analysis}

All results are showed as mean \pm SEM. When compared two groups, an unpaired Student's t test was used. When compared four groups, one-way ANOVA followed by Bonferroni post hoc test was used. Twoway ANOVA was used to analyze MPWT, NORT. GraphPad Prism 7.0 was used for statistical analysis, and $P<0.05$ as a statistically significant $p$ value.

\section{Results}

\section{SNI induced mechanical allodynia, hippocampus-dependent memory impairment and decreased GLP- 1 andGLP-1R in mice}

To evaluate development of mechanical allodynia after SNI surgery, the MPWT was tested on day 0 (baseline), day 3,day 7,day 10,day 14,day 21 after the surgeries(Fig. 1B). The results showed that MPWT was significantly decreased in SNI mice, compared to sham mice.

Next, we evaluated the novel-object recognition memory via the NORT. In the NORT, no significant difference was found in the total time spent on identical objects between the Sham and SNI groups during the training phase (Fig. 1C). In the testing stage, the mice spent more time on the novel object than on the familiar object in the Sham group (Fig. 1D). However, the time spent on the novel and familiar objects was similar in the SNI group. Furthermore, the discrimination ratio in the Sham group was higher than that in the SNI group (Fig. 1E).These results demonstrate that recognition memory was impaired in the mice with neuropathic pain.

In addition to this, we uncovered that the GLP-1/GLP-1R axis was inhibited in the murine hippocampus induced by neuropathic pain (Fig. 1F, G). West Blot showed that the levels of GLP-1 and GLP-1R were decreased in murine hippocampus.

\section{SNI caused disturbance of inflammatory signaling, neuroinflammation, and decreased synaptic associated proteins in the murine hippocampus}

We found that the AMPK/NF-KB signaling was disturbed in the murine hippocampus with cognitive dysfunction. Neuropathic pain could inhibit the activation of AMPK by inhibiting its phosphorylation at the Thr172 site (Fig. 2A) but activate its downstream NF-KB p65 by increasing the level of p- NF-KB p65 (Fig. 2B) compared with the Sham group. Furthermore, activating NF-kB p65 could induce neuroinflammation by up-regulating the expression of pro-inflammatory cytokines, including mature IL-1 $\beta$ (IL-1 $\beta$ p17) (Fig. 2C) in the hippocampus of mice. Also, over expressed IL-1 $\beta$ could decrease the level of synaptic associated proteins, such as PSD95 (Fig. 2D) and Arc (Fig. 2E), thus inducing memory impairment, compared with Sham mice. 


\section{EX-4, a GLP-1R agonists, improved memory impairment caused by SNI in mice}

First, we evaluated the effects of Ex-4 on mechanical allodynia and memory deficit in mice with neuropathic pain after SNI. In the MPWT test, we found that both vehicle and Ex-4 application had no effect on allodynia in the SNI mice compared with that in the Sham + Veh and Sham + Ex-4 groups (Fig. 3B).

In the training phase of the NORT, no difference was found in the total time spent on identical objects among the four groups (Fig. $3 \mathrm{C}$ ). In the testing stage, the mice spent more time on the novel object than on the familiar object in the Sham + Veh, Sham + Ex-4 and SNI + Ex-4groups. However, no difference was found in the time spent with novel and familiar objects in the SNI + Veh group(Fig. 3D). In addition, the DR in the SNI + Veh group was lower than that in the Sham + Veh group, but Ex-4 application could increase the DR in SNI mice compared with that in $\mathrm{SNI}+$ Veh mice(Fig. 3E).Taken together, these results suggested that Ex-4 can ameliorate deficits in the recognition memory caused by neuropathic pain.

\section{Ex-4 improved inflammatory signaling, neuroinflammation, and upregulated synaptic proteins in mice hippocampus}

Next, we explored whether Ex-4 treatment could regulate the inflammatory signaling pathway and improve inflammation, the decreased synaptic associated-proteins. It was found that, in the murine hippocampus with memory deficits, Ex-4 could reverse the down-regulated levels of p-AMPKThr172 compared with Veh group (Fig. 4A), and could also inhibit the phosphorylation of NF-kB p65 downstream of AMPK (Fig. 4B).Furthermore, Ex-4 could attenuate neuroinflammation by down-regulating the secretion of IL-1 $\beta$ p17 (Fig. 4C). The results suggested that Ex-4 restored the decreased levels of PSD95 (Fig. 4D) and Arc (Fig. 4E) in the murine hippocampus, which was consistent with the memory protection of EX-4.

\section{Ex(9-39),a GLP-1R antagonist, reversed the memory protection of Ex-4 in SNI mice}

We further verified the memory protective effect of activating GLP-1R in SNI mice via administration with Ex-4 and Ex(9-39).In the MPWT , the results showed both Ex-(9-39) and Ex-4 + Ex(9-39) treatment did not affect the decrease of MPWT in SNI mice compared with that in the SNI + Veh group(Fig. 5B).In the NORT, the four groups spent a similar total time exploring two identical objects during the training phase(Fig. 5C). However, in the testing stage, the SNI + Ex-4 + Veh group spent more time on the novel object than on the familiar object compared with the SNI + Veh and SNI + Ex-4 + Ex(9-39) groups(Fig. 5D).It was found that $\operatorname{Ex}(9-39)$ could abrogate the increase in DR induced by Ex-4 in mice(Fig. 5E).

\section{Ex(9-39) abolished the anti-inflammatory effect of EX-4 in thehippocampus of SNI mice}

First, we reported that Ex-4 could activate AMPK by increasing the level of p-AMPKThr172, however, Ex(939) could inhibit the activation of AMPK induced by Ex-4 in SNI mice(Fig. 6A). Similarly, Ex(9-39) could also upregulate the decreased level of p-NF-KBp65 downstream of AMPK caused by Ex-4 in SNI mice(Fig. 6B).Moreover, Ex(9-39) could reverse the anti-inflammatory effect of Ex-4 by promoting the 
expression of IL-1 $\beta$ p17 (Fig. 6C).We also uncovered that $\operatorname{Ex}(9-39)$ could reversed the increased synaptic associated proteins PDS95 (Fig. 6D) and Arc (Fig. 6E) induced by Ex-4 in mice with memory impairment caused by neuropathic pain.

\section{CC, an AMPK inhibitor, reversed improved recognition memory caused by Ex-4 in SNI mice}

In order to further explore the mechanism of activating GLP-1R to improve recognition memory dysfunction caused by neuropathic pain, we applied Ex-4 and CC in SNI mice. We found that both CC and Ex-4 + CC did not influence the MPWT in SNI mice as compared to that in the SNI + Veh mice (Fig. 7B). In the NORT, during the training phase, no significant difference was found in the total time spent on identical objects among the four groups (Fig. 7C). However, the SNI + Ex-4 group spent significantly more time on the novel object than on the familiar object compared to the $\mathrm{SNI}+$ Veh or SNI + Ex-4 + CC groups(Fig. 7D).About DR, the results demonstrated that application with $\mathrm{CC}$ could reverse the increased the DR induced by Ex-4 in SNI mice (Fig. 7E).

\section{CC weakened the neuroprotection of Ex-4 in SNI mice}

We used CC to verify whether the improved recognition memory effect via activation of GLP-1R was mediated by regulating the AMPK/NF-KB signaling pathway. We discovered that activating GLP-1R could inhibit the activation of NF-KB via AMPK. On the contrary, when an AMPK inhibitor was administered in mice, it was found that the protein level of p- NF-KB p65 in the SNI+Ex-4+CC group was significantly higher than that in the SNI+Ex-4+Veh group(Fig 8A).Furthermore, activation of GLP-1R by Ex-4 treatment in the mice with memory dysfunction induced by SNI could ameliorate neuroinflammation, but CC could abolish this effect (Fig 8B). We also tested synaptic-associated proteins, the results suggested that the combined application of Ex-4 and CC could downregulate the contents of PSD95(Fig 8C) and Arc(Fig 8D) proteins compared that of Ex-4 alone in SNI mice. The results suggested that activating GLP-1R might rescue the decreased level of synaptic associated proteins via improving neuroinflammation through regulation of AMPK/NF-KB signaling.

\section{Discussion}

The major findings of this research were as follows: first, neuropathic pain can impair recognition memory and the level of GLP-1R was decreased in the hippocampus of mice with memory impairment. Second, the activation of GLP-1R could improve memory impairments caused by neuropathic pain. Third, the function of Ex-4 of improving memory deficits induced by chronic pain of Ex-4 was reversed by GLP$1 \mathrm{R}$ antagonists. Fourth, AMPK inhibitors abrogated the neuroprotection of GLP-1R in mice with neuropathic pain. Taken together, it was likely that hippocampal GLP-1R played a critical role in protecting memory from neuropathic pain by downregulating the expression level of IL-1 $\beta$ by regulating the AMPK/NF-KB signaling pathway.

Previous studies have shown that the activation of GLP-1R could improve impaired learning and memory function $[6,24-26]$. It was detected that GLP-1 and GLP-1R were both decreased in AD human brain and 
APP/PS1/Tau transgenic (3xTg) murine brains [26]. However, improving the level of GLP-1and GLP-1R expression by dipeptidyl peptidase-4 (DPP-4) inhibitors in the hippocampus and cortex of AD mice brains could improve impaired learning and memory, increase the 0-Glycosylation and synaptic proteins, and decrease abnormal phosphorylation of tau and neurofilaments (NFs) and intercellular $\beta$-amyloid (A $\beta$ ) accumulation[26]. In this study, we showed that the GLP-1/GLP-1R axis was repressed in mice with memory impairment induced by neuropathic pain. We also found that Ex-4 treatment $(0.4 \mathrm{mg} / \mathrm{kg}, \mathrm{s} . \mathrm{c}$.) did not ameliorate pain hypersensitivity induced by SNI. However, Gong et al. reported that intrathecal exenatide potently alleviated peripheral nerve injury (tight ligation of peripheral L5-L6 spinal nerves) induced hypersensitivity states by $60-90 \%$ in a dose and time-dependent antinociception way [27]. It is possible that the dose of Ex-4 in our experiment did not reach the concentration which could suppress pain hypersensitivity via activation of GLP-1R in the spinal cord.

AMPK is a critical sensor of energy status, which regulates cellular energy homeostasis[28]. Several studies have shown that activating AMPK could ameliorate inflammation by inhibiting the activation of NF-KB $[13,14,29,30]$.Furthermore, it has been reported that AMPK could be activated as a downstream of GLP-1R,when GLP-1R is activated[10-12, 31]. Stimulating GLP-1R using sitagliptin could facilitate axonal regeneration and locomotor functional repair after spinal cord injury via GLP-1R induced AMPK/PGC-1a signaling pathway activation [12]. A certain study has demonstrated that GLP-1 may suppress hepatic fat accumulation and inflammatory response induced by nutrients via the activation of the AMPK pathway [31].In our study, we demonstrated that AMPK was suppressed; however, NF-KB was hyperactive in mice with memory deficits caused by neuropathic pain. Nevertheless, the activation of GLP-1R could reverse the disturbance of AMPK/NF-KB signaling in the murine hippocampus induced by neuropathic pain.

It is well known that neuroinflammation is a common cause of memory deficits, and neuropathic pain often promotes the secretion of pro-inflammatory mediators in the murine hippocampus. The level of TNF-a in the bilateral hippocampus was increased, which could impair LTP in the hippocampus and reduce the dendrite lengths and spine densities in hippocampal CA1 pyramidal neurons [3]. However, it was found that the activation of GLP-1R could ameliorate neuroinflammation [7, 8, 18, 32]. The activation of GLP-1R by Ex-4 could reduce the transcriptional expression level of pro-inflammatory cytokines (IL-1 $\beta$, IL-6, IL-17, TNF-a) in the injured spinal cords of experimental autoimmune encephalomyelitis (EAE) mice [18]. Besides this, it was found that activating GLP-1R using Ex-4 could rescue surgery-induced spatial learning and memory deficits via inhibiting NF-KB signaling and reducing the expression of IL-1 [32]. In our study, we found that the activation of GLP-1R could reduce the secretion of mature IL-1 $\beta$ in murine hippocampus induced by neuropathic pain.

PSD95 is a member of the membrane-associated guanylate kinase (MAGUK) family, which can interact with certain neurotransmitter receptors, including N-methyl-D-aspartate (NMDA) receptors [33], and aamino-3-hydroxyl-5-methyl-4-isoxazolepropionic acid (AMPA) receptors [34], and participate in synaptic plasticity and synaptic stabilization in LTP [35]. Arc is a protein that modulates the subsynaptic cytoskeleton and LTP [36], which contributes to memory consolidation and represents the activation of neurons during learning. Also, Arc participates in multiple synaptic plasticities mediated by the AMPA 
receptors [37].Furthermore, the overexpression of IL-1 $\beta$ might downregulate the expression of PSD95 [38] and Arc [36], which contributes to memory impairment. In this study, we found that neuropathic pain decreased the contents of PSD95 and Arc in the hippocampus of mice with memory impairment caused by neuropathic pain, but activating GLP-1R could upregulate the protein levels of PSD95 and Arc.

\section{Conclusions}

In short, the current study demonstrated that GLP-1R plays a critical role in improving neuropathic paininduced novel object recognition memory dysfunction. Importantly, we found that the expression of GLP$1 \mathrm{R}$ was downregulated in the murine hippocampus with recognition memory dysfunction, which exacerbated neuropathic pain-induced recognition memory deficits. Moreover, the activation of GLP-1R by Ex-4 could reverse recognition memory dysfunction by regulating the AMPK/NF-KB signaling pathway, which could ameliorate neuroinflammation and restore the decreased expression of synaptic proteins. Therefore, GLP-1R might as a potential target for improving memory impairment induced by neuropathic pain.

\section{Abbreviations}

GLP-1: Glucagon-like-peptide-1; GLP-1R: Glucagon-like-peptide-1receptor; AMPK: adenosine monophosphate-activated protein kinase; NF-kB: nuclear factor K B; IL-1 $\beta$ : interleukin-1 $\beta$; PSD95: postsynaptic density protein 95 ; Arc: activity-regulated cytoskeleton-associated protein; Ex-4: exenatide acetate; Ex(9-39): exendin(9-39); CC: Compound C dihydrochloride

\section{Declarations}

\section{Acknowledgments}

We thank all colleagues for their contributions to this research.

\section{Authors' contributions}

XBT conceived the project and supervised all experiments. LQZ wrote the manuscript. LQZ, ZW, TL performed work on behavioral tests. LQZ, TY, XMY, YQZ, QZ performed work on western blot. FG and HY performed work on drug administration. YKT and WM performed work on statistical analysis. All authors read and approved the final manuscript.

\section{Funding}

This work was financially supported by grants from the National Natural Science Foundation of China 81974170.

\section{Availability of data and materials}


All data generated or analyzed during this study are included in this article

Ethics approval and consent to participate

All experiments were approved by the Experimental Animal Care and Use Committee of Tongji hospital,

Tongji Medical College, Huazhong University of Science and Technology.

Consent for publication

Not applicable

Competing interests

The authors declare that they have no competing interests

\section{References}

1. Xiong B, Zhang W, Zhang L, Huang X, Zhou W, Zou Q, Manyande A, Wang J, Tian Y, Tian X: Hippocampal glutamatergic synapses impairment mediated novel-object recognition dysfunction of neuropathic pain in rats. Pain 2020.

2. Ren WJ, Liu Y, Zhou LJ, Li W, Zhong Y, Pang RP, Xin WJ, Wei XH, Wang J, Zhu HQ, et al: Peripheral nerve injury leads to working memory deficits and dysfunction of the hippocampus by upregulation of TNF-alpha in rodents. Neuropsychopharmacology 2011, 36:979-992.

3. Liu Y, Zhou LJ, Wang J, Li D, Ren WJ, Peng J, Wei X, Xu T, Xin WJ, Pang RP, et al: TNF-alpha Differentially Regulates Synaptic Plasticity in the Hippocampus and Spinal Cord by MicrogliaDependent Mechanisms after Peripheral Nerve Injury. J Neurosci 2017, 37:871-881.

4. You Z, Zhang S, Shen S, Yang J, Ding W, Yang L, Lim G, Doheny JT, Tate S, Chen L, Mao J: Cognitive impairment in a rat model of neuropathic pain: role of hippocampal microtubule stability. Pain 2018, 159:1518-1528.

5. Cork SC, Richards JE, Holt MK, Gribble FM, Reimann F, Trapp S: Distribution and characterisation of Glucagon-like peptide-1 receptor expressing cells in the mouse brain. Mol Metab 2015, 4:718-731.

6. During MJ, Cao L, Zuzga DS, Francis JS, Fitzsimons HL, Jiao X, Bland RJ, Klugmann M, Banks WA, Drucker DJ, Haile CN: Glucagon-like peptide-1 receptor is involved in learning and neuroprotection. Nat Med 2003, 9:1173-1179.

7. Gullo F, Ceriani M, D'Aloia A, Wanke E, Constanti A, Costa B, Lecchi M: Plant Polyphenols and Exendin-4 Prevent Hyperactivity and TNF-alpha Release in LPS-Treated In vitro Neuron/Astrocyte/Microglial Networks. Front Neurosci 2017, 11:500.

8. Diz-Chaves Y, Toba L, Fandino J, Gonzalez-Matias LC, Garcia-Segura LM, Mallo F: The GLP-1 analog, liraglutide prevents the increase of proinflammatory mediators in the hippocampus of male rat pups submitted to maternal perinatal food restriction. J Neuroinflammation 2018, 15:337. 
9. Zhang W, Xiong BR, Zhang LQ, Huang X, Zhou WC, Zou Q, Manyande A, Wang J, Tian XB, Tian YK: Disruption of the GABAergic system contributes to the development of perioperative neurocognitive disorders after anesthesia and surgery in aged mice. CNS Neurosci Ther 2020.

10. He Q, Sha S, Sun L, Zhang J, Dong M: GLP-1 analogue improves hepatic lipid accumulation by inducing autophagy via AMPK/mTOR pathway. Biochem Biophys Res Commun 2016, 476:196-203.

11. Ma ZG, Kong CY, Song P, Zhang X, Yuan YP, Tang QZ: Geniposide Protects against Obesity-Related Cardiac Injury through AMPKalpha- and Sirt1-Dependent Mechanisms. Oxid Med Cell Longev 2018, 2018:6053727.

12. Han W, Li Y, Cheng J, Zhang J, Chen D, Fang M, Xiang G, Wu Y, Zhang H, Xu K, et al: Sitagliptin improves functional recovery via GLP-1R-induced anti-apoptosis and facilitation of axonal regeneration after spinal cord injury. J Cell Mol Med 2020.

13. Nunes AK, Raposo C, Rocha SW, Barbosa KP, Luna RL, da Cruz-Hofling MA, Peixoto CA: Involvement of AMPK, IKbetaalpha-NFkappaB and eNOS in the sildenafil anti-inflammatory mechanism in a demyelination model. Brain Res 2015, 1627:119-133.

14. Xiang HC, Lin LX, Hu XF, Zhu H, Li HP, Zhang RY, Hu L, Liu WT, Zhao YL, Shu Y, et al: AMPK activation attenuates inflammatory pain through inhibiting NF-kappaB activation and IL-1beta expression. $J$ Neuroinflammation 2019, 16:34.

15. Wang L, Yin C, Liu T, Abdul M, Zhou Y, Cao J-L, Lu C: Pellino1 regulates neuropathic pain as well as microglial activation through the regulation of MAPK/NF-KB signaling in the spinal cord. Journal of Neuroinflammation 2020, 17.

16. Gui WS, Wei X, Mai CL, Murugan M, Wu LJ, Xin WJ, Zhou LJ, Liu XG: Interleukin-1beta overproduction is a common cause for neuropathic pain, memory deficit, and depression following peripheral nerve injury in rodents. Mol Pain 2016, 12.

17. Mai CL, Wei X, Gui WS, Xu YN, Zhang J, Lin ZJ, Tan Z, Meng YT, Li YY, Zhou LJ, Liu XG: Differential regulation of GSK-3beta in spinal dorsal horn and in hippocampus mediated by interleukin-1beta contributes to pain hypersensitivity and memory deficits following peripheral nerve injury. Mol Pain 2019, 15:1744806919826789.

18. Lee CH, Jeon SJ, Cho KS, Moon E, Sapkota A, Jun HS, Ryu JH, Choi JW: Activation of Glucagon-Like Peptide-1 Receptor Promotes Neuroprotection in Experimental Autoimmune Encephalomyelitis by Reducing Neuroinflammatory Responses. Mol Neurobio/ 2018, 55:3007-3020.

19. Decosterd. I, Woolf. CJ: Spared nerve injury an animal model of persistent peripheral neuropathic pain. PAIN 2000, 87:149-158.

20. Bourquin AF, Suveges M, Pertin M, Gilliard N, Sardy S, Davison AC, Spahn DR, Decosterd I: Assessment and analysis of mechanical allodynia-like behavior induced by spared nerve injury (SNI) in the mouse. Pain 2006, 122:14 e11-14.

21. An J, Zhou Y, Zhang M, Xie Y, Ke S, Liu L, Pan X, Chen Z: Exenatide alleviates mitochondrial dysfunction and cognitive impairment in the 5XFAD mouse model of Alzheimer's disease. Behav Brain Res 2019, 370:111932. 
22. Day SM, Yang W, Ewin S, Zhou X, Ma T: Glucagon-like peptide-1 cleavage product GLP-1 (9-36) amide enhances hippocampal long-term synaptic plasticity in correlation with suppression of Kv4.2 expression and eEF2 phosphorylation. Hippocampus 2017, 27:1264-1274.

23. Shen P, Hou S, Zhu M, Zhao M, Ouyang Y, Feng J: Cortical spreading depression preconditioning mediates neuroprotection against ischemic stroke by inducing AMP-activated protein kinasedependent autophagy in a rat cerebral ischemic/reperfusion injury model. J Neurochem 2017, 140:799-813.

24. Li Y, Bader M, Tamargo I, Rubovitch V, Tweedie D, Pick CG, Greig NH: Liraglutide is neurotrophic and neuroprotective in neuronal cultures and mitigates mild traumatic brain injury in mice. $J$ Neurochem 2015, 135:1203-1217.

25. Cai HY, Yang JT, Wang ZJ, Zhang J, Yang W, Wu MN, Qi JS: Lixisenatide reduces amyloid plaques, neurofibrillary tangles and neuroinflammation in an APP/PS1/tau mouse model of Alzheimer's disease. Biochem Biophys Res Commun 2018, 495:1034-1040.

26. Chen S, Zhou M, Sun J, Guo A, Fernando RL, Chen Y, Peng P, Zhao G, Deng Y: DPP-4 inhibitor improves learning and memory deficits and AD-like neurodegeneration by modulating the GLP-1 signaling. Neuropharmacology 2019, 157:107668.

27. Gong N, Xiao Q, Zhu B, Zhang CY, Wang YC, Fan H, Ma AN, Wang YX: Activation of spinal glucagonlike peptide-1 receptors specifically suppresses pain hypersensitivity. J Neurosci 2014, 34:5322-5334.

28. Hardie DG: AMP-activated protein kinase: an energy sensor that regulates all aspects of cell function. Genes Dev 2011, 25:1895-1908.

29. Jian M, Kwan JS, Bunting M, Ng RC, Chan KH: Adiponectin suppresses amyloid-beta oligomer (Abeta0)-induced inflammatory response of microglia via AdipoR1-AMPK-NF-kappaB signaling pathway. J Neuroinflammation 2019, 16:110.

30. Hu R, Wang MQ, Ni SH, Wang M, Liu LY, You HY, Wu XH, Wang YJ, Lu L, Wei LB: Salidroside ameliorates endothelial inflammation and oxidative stress by regulating the AMPK/NFkappaB/NLRP3 signaling pathway in AGEs-induced HUVECs. Eur J Pharmacol 2020, 867:172797.

31. Ben-Shlomo S, Zvibel I, Shnell M, Shlomai A, Chepurko E, Halpern Z, Barzilai N, Oren R, Fishman S: Glucagon-like peptide-1 reduces hepatic lipogenesis via activation of AMP-activated protein kinase. J Hepatol 2011, 54:1214-1223.

32. Zhou Y, Li Z, Cao X, Ma H, White PF, Xu X, Jiang Y, Sun X, Cui Y: Exendin-4 improves behaviorial deficits via GLP-1/GLP-1R signaling following partial hepatectomy. Brain Res 2019, 1706:116-124.

33. Won S, Incontro S, Nicoll RA, Roche KW: PSD-95 stabilizes NMDA receptors by inducing the degradation of STEP61. Proc Natl Acad Sci U S A 2016, 113:E4736-4744.

34. Colledge M, Snyder EM, Crozier RA, Soderling JA, Jin Y, Langeberg LK, Lu H, Bear MF, Scott JD: Ubiquitination regulates PSD-95 degradation and AMPA receptor surface expression. Neuron 2003, 40:595-607.

35. Meyer D, Bonhoeffer T, Scheuss V: Balance and stability of synaptic structures during synaptic plasticity. Neuron 2014, 82:430-443. 
36. Tong L, Prieto GA, Kramar EA, Smith ED, Cribbs DH, Lynch G, Cotman CW: Brain-derived neurotrophic factor-dependent synaptic plasticity is suppressed by interleukin-1beta via p38 mitogen-activated protein kinase. J Neurosci 2012, 32:17714-17724.

37. Mabb AM, Ehlers MD: Arc ubiquitination in synaptic plasticity. Semin Cell Dev Bio/2018, 77:10-16.

38. Dong Y, Li S, Lu Y, Li X, Liao Y, Peng Z, Li Y, Hou L, Yuan Z, Cheng J: Stress-induced NLRP3 inflammasome activation negatively regulates fear memory in mice. $J$ Neuroinflammation 2020 , 17:205.

\section{Figures}



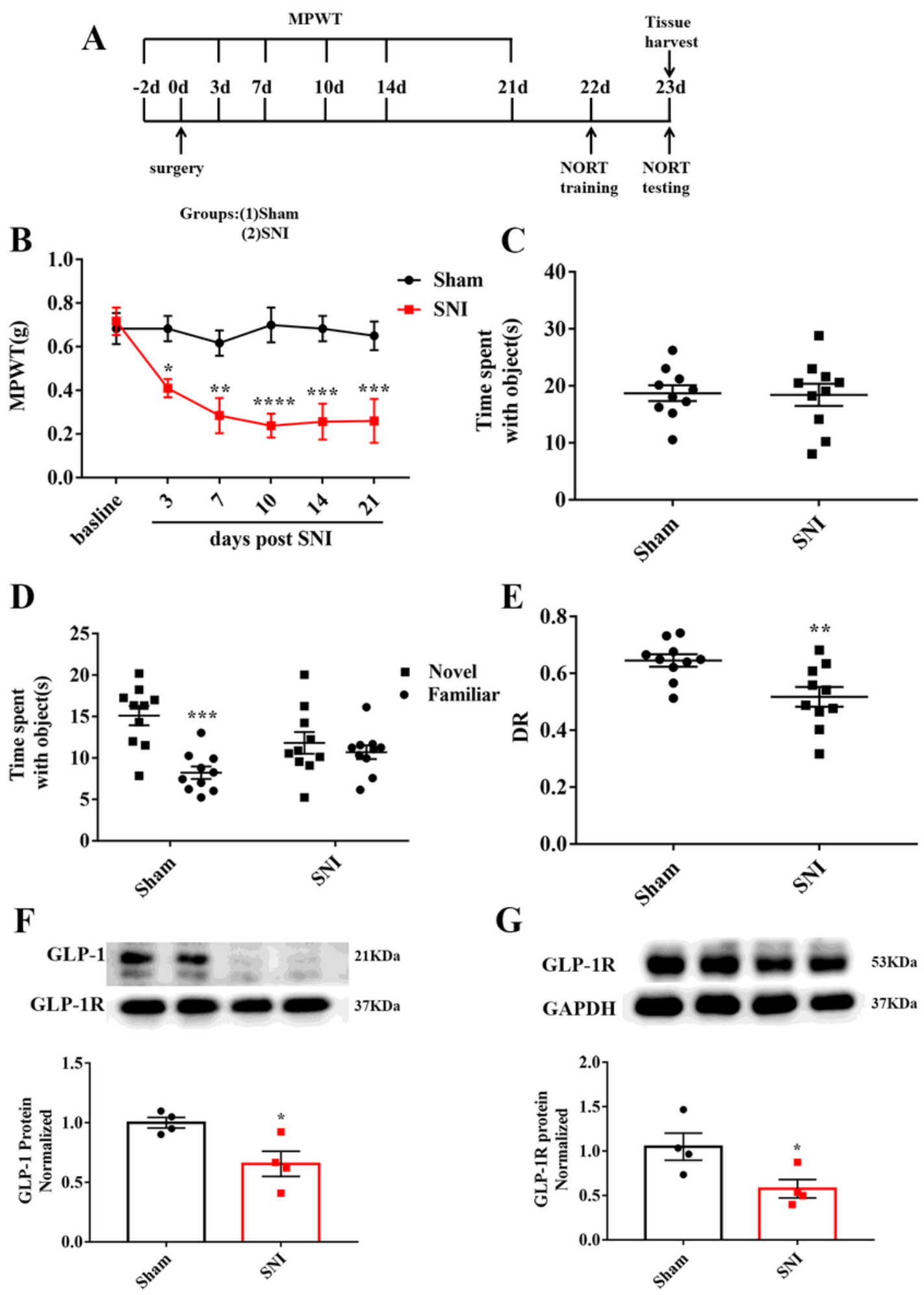

Figure 1

SNI induced the decrease of MPWT, impaired recognition memory impairment and suppressed the GLP1/GLP-1R signaling axis. a The diagram shows the process of this experiment. The MPWT was measured 2 days before surgery as the baseline, and MPWTs were also tested $3,7,10,14$, and 21 days after SNI. The NORT was tested 22 and 23 days after SNI. The hippocampus was collected after NORT. b The ipsilateral MPWT was significantly decreased from day 3 to day 21 after surgery in SNI mice compared 
with sham group ( $\mathrm{n}=12$ mice per group). $\mathrm{c}$ In the NORT training section, there were no significant differences in time spent on exploring two identical objects (familiar object) between the Sham and SNI groups(n=10 mice per group). $d$ In the NORT testing phase, sham mice spent more time exploring novel objects rather than familiar objects compared to the $S N I$ group( $n=10$ mice per group). e DR represented recognition memory; the SNI mice demonstrated a significant reduction in DR compared with the sham group( $n=10$ mice per group). $f-g$ Neuropathic pain dramatically downregulated the protein expression

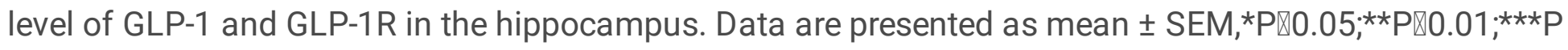
$\llbracket 0.001 ; * \star \star * P \otimes 0.0001$, compared with the sham group. 
A

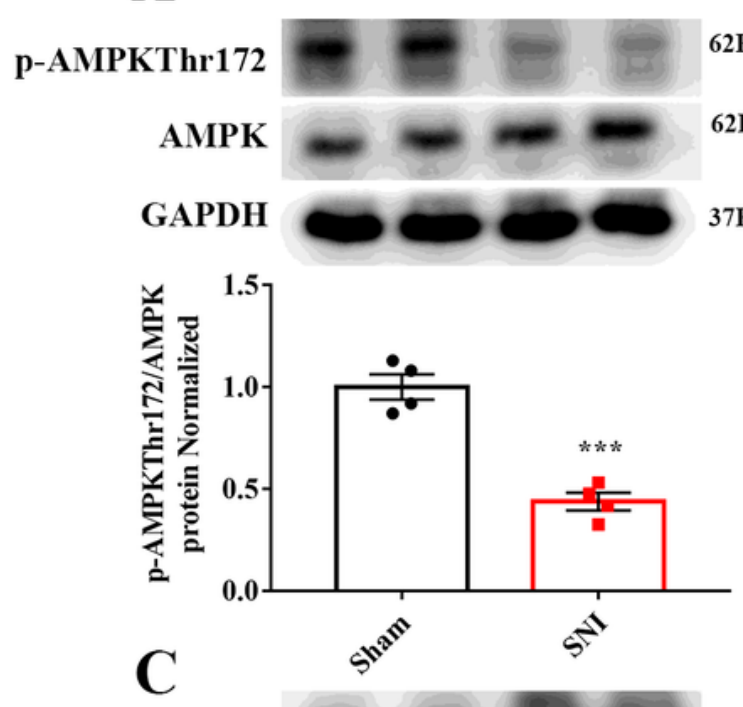

IL-1beta p17 $-0-000$ 17KDa
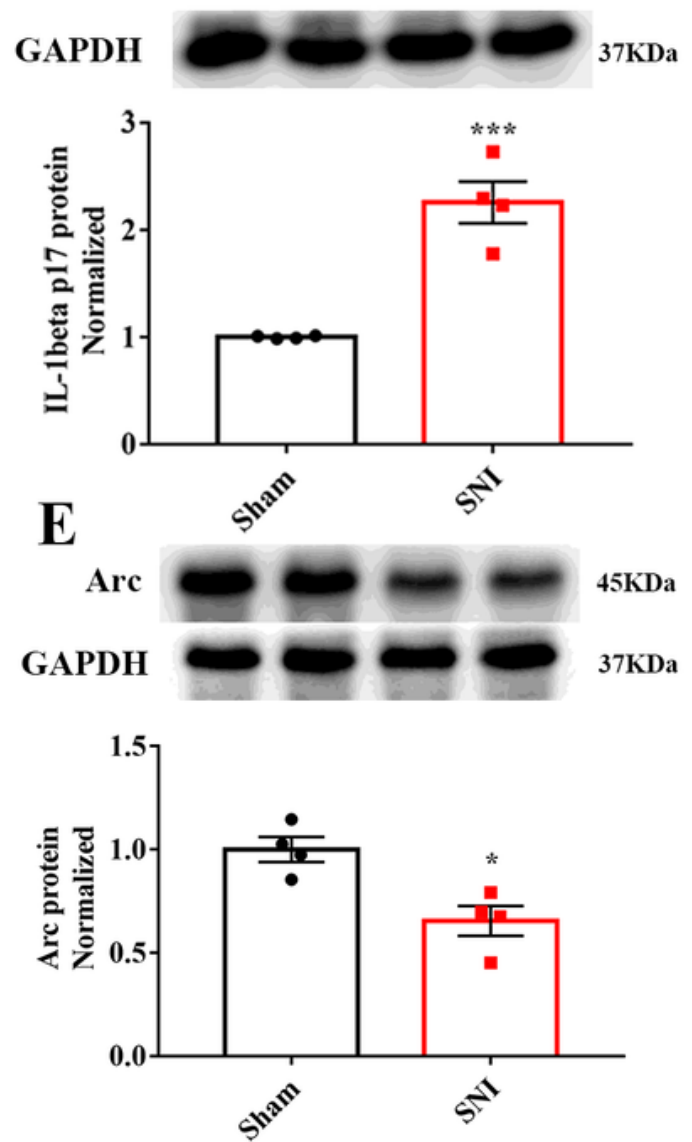

B

$\mathrm{p}-\mathrm{NF}-\kappa \mathrm{B}$ p65 we $=100$

NF-кB p65 $=-\infty 5 \mathrm{KDa}$

GAPDH $\longrightarrow$ 37KDa

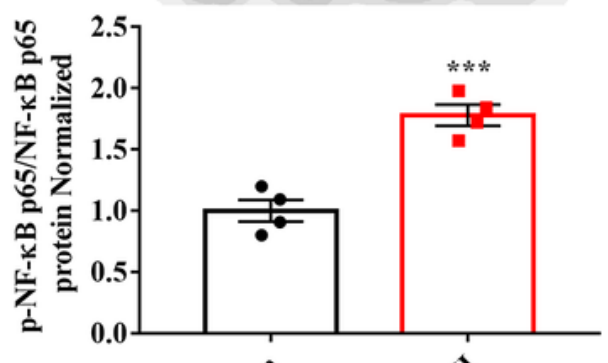

D के के

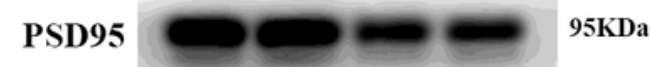

GAPDH $\longrightarrow$ 37KDa

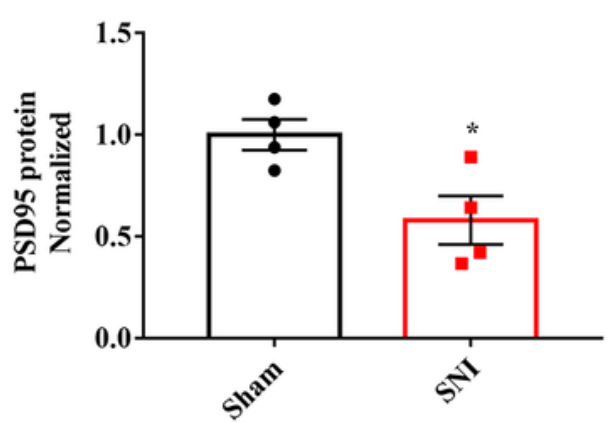

\section{Figure 2}

Neuropathic pain disturbed AMPK/NF-KB signaling, induced neuroinflammation and downregulated the expression level of synaptic proteins in mice hippocampus. a-b SNI could inhibit the phosphorylation of AMPK and promote the phosphorylation of NF-KB p65 in the hippocampus compared with the sham group ( $n=4$ mice per group). c Neuropathic pain remarkably increased the level of IL-1 $\beta$ p17 in mice with memory deficits ( $n=4$ mice per group). $d$-e Neuropathic pain impaired recognition memory by 
downregulating the level of synaptic associated proteins ( $n=4$ mice per group).Data are presented as mean $\pm S E M, * P<0.05 ; * \star * P<0.001$, compared to the sham group.

A

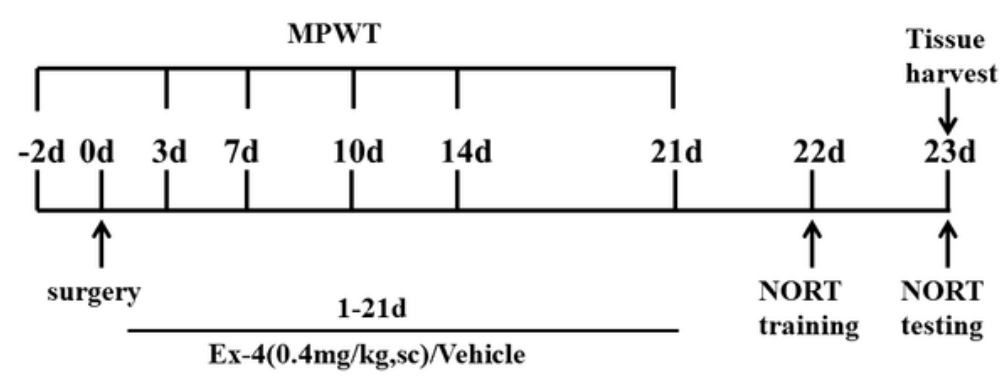

Groups:(1)Sham+Vehicle

(2)Sham+Ex-4

(3)SNI+Vehicle

(4) SNI+Ex-4
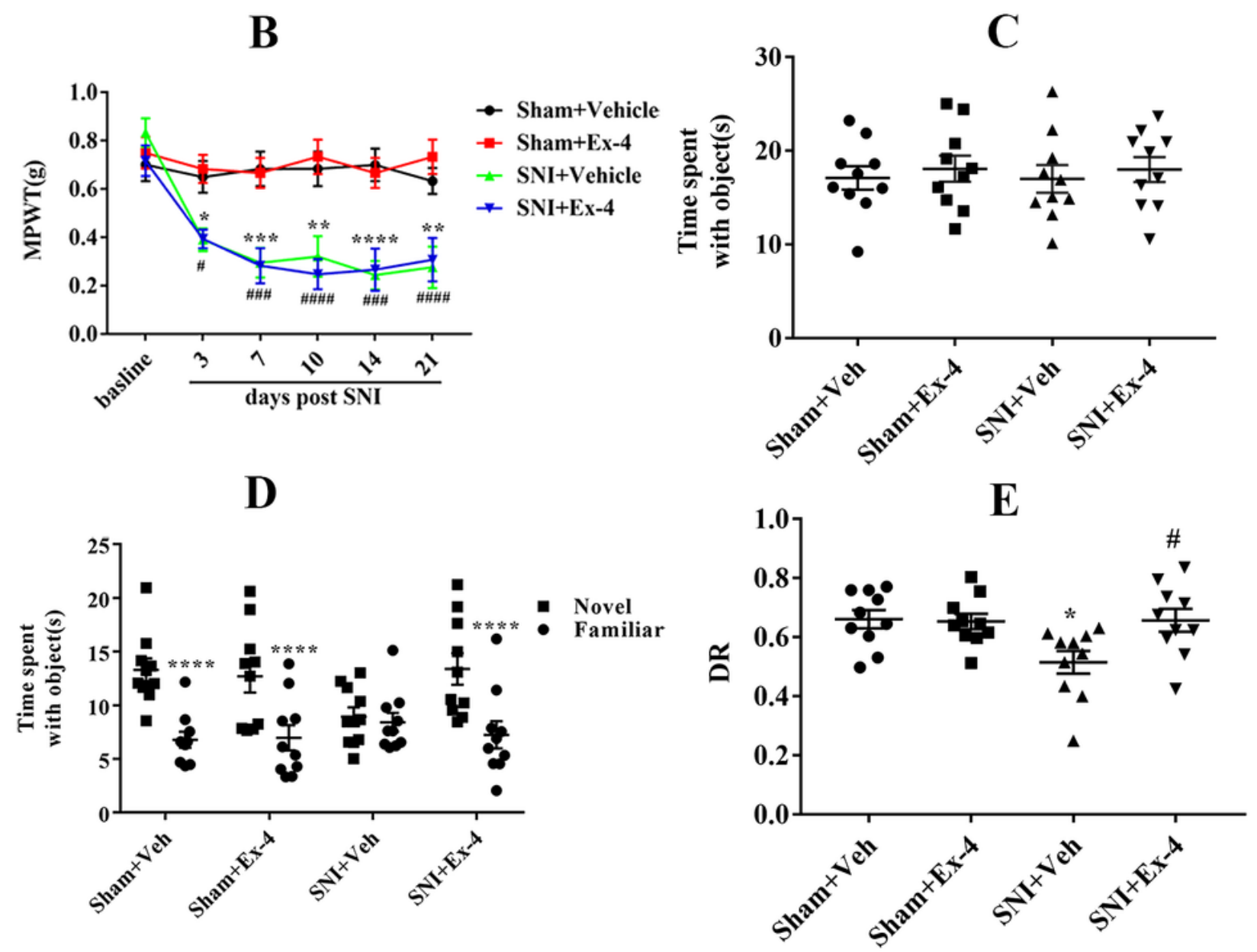

Figure 3

Ex-4 treatment could reverse the recognition memory dysfunction caused by neuropathic pain. a The schedule of administering Ex-4 or vehicle, MPWT and NORT.Ex- $4(0.4 \mathrm{mg} / \mathrm{kg}$, s.c.) or an equal volume of saline was administered continuously for 21 days both in the sham and SNI groups, starting from the first 
day after the operation. $b$ Both the Ex-4 and vehicle treatments induced a decrease in MPWT.Ex-4 treatment did not reverse the allodynia induced by nerve injury compared with the Sham+Ex-4 group ( $n=12$ mice per group). $c$ In the NORT training phase, there were no significant differences in time spent on exploration two identical objects (familiar object) between the four groups ( $n=10$ mice per group). $d$ In the NORT testing phase, Ex-4 treatment demonstrated a significant increase in exploration time of the novel object compared with the vehicle group in SNI mice ( $n=10$ mice per group). e Compared with that in the Sham+Veh group, neuropathic pain decreased DR.However,Ex-4 could reverse the decreased DR compared with $\mathrm{SNI}+\mathrm{Veh}$ group ( $\mathrm{n}=10$ mice per group). Data are presented as mean \pm $S E M,{ }^{*} P<0.05 ; * \star P<0.01 ; * \star * P<0.001 ; * \star * \star P<0.0001 ; \# P<0.05 ; \# \# \# P<0.001 ; \# \# \# \# P<0.0001$. 
A

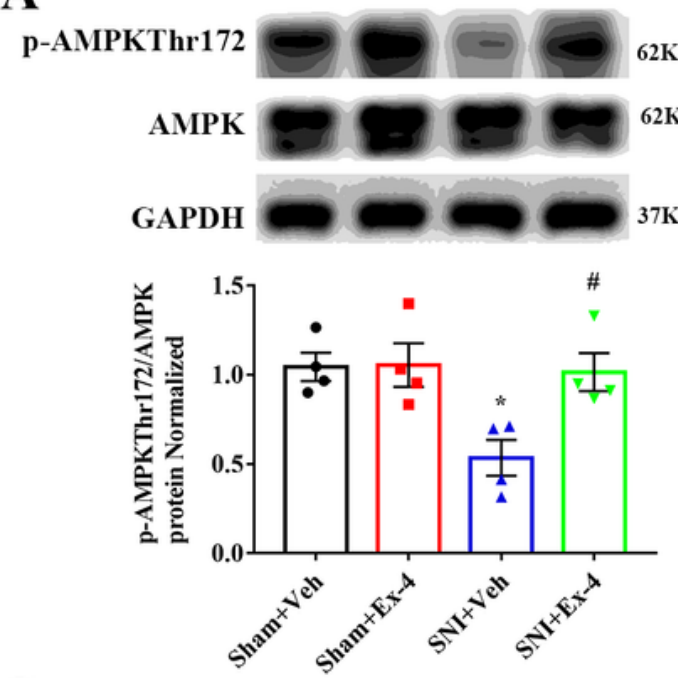

C
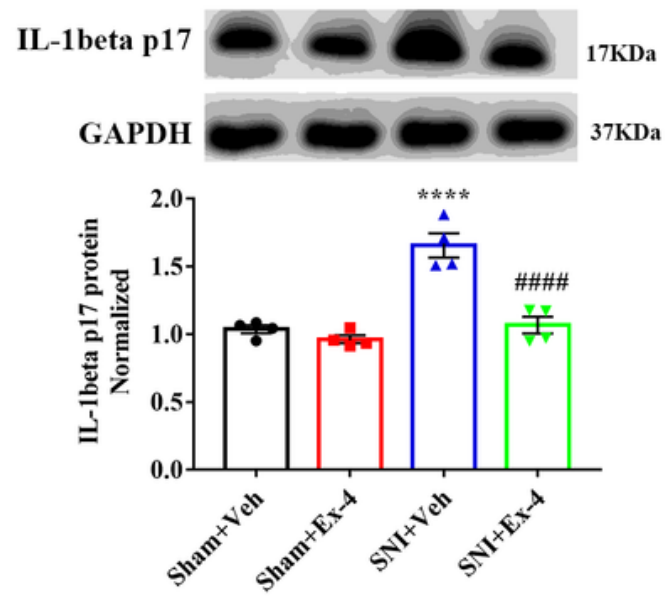

E

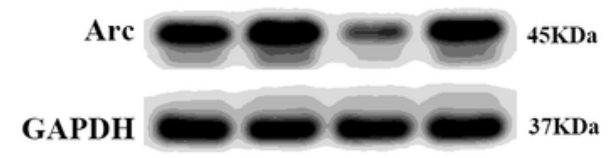

B
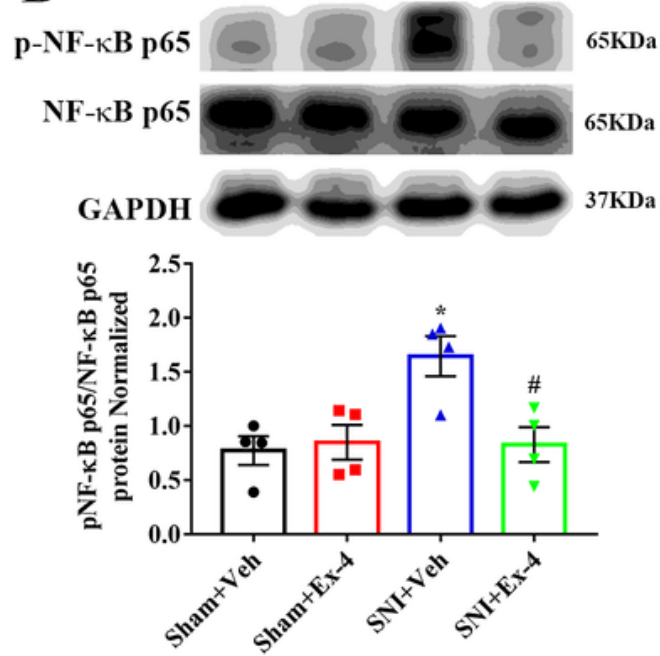

D
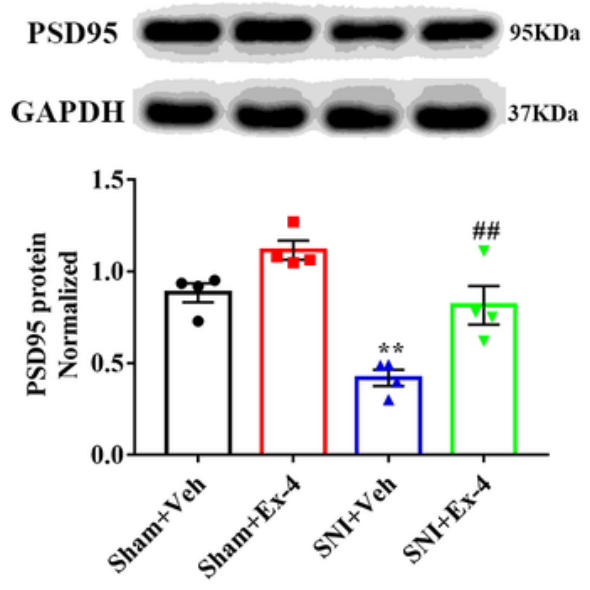

Figure 4

Administration with Ex-4 could improve the unbalanced AMPK/NF-KB signaling pathway, neuroinflammation and upregulate the level of synaptic proteins in mice with memory impairment. a-b neuropathic pain decreased the expression level of p-AMPKThr172 and increased p-NF-KB p65; however, application with Ex-4 could reverse the effects ( $n=4$ mice per group). c Compared to the SNI+Veh group, Ex-4 treatment could significantly reduce the protein level of IL-1 $\beta$ p17 ( $n=4$ mice per group). $d-e$ Ex-4 
treatment abolished the decrease of synaptic proteins in mice with memory dysfunction induced by neuropathic pain ( $n=4$ mice per group). Data are presented as mean $\pm S E M,{ }^{*} P<0.05 ;{ }^{*} P<0.01$; ****P<0.0001; $\# \mathrm{P}<0.05 ; \# \# \mathrm{P}<0.01: \# \# \mathrm{P}<0.001 ; \# \# \# \# \mathrm{P}<0.0001$.

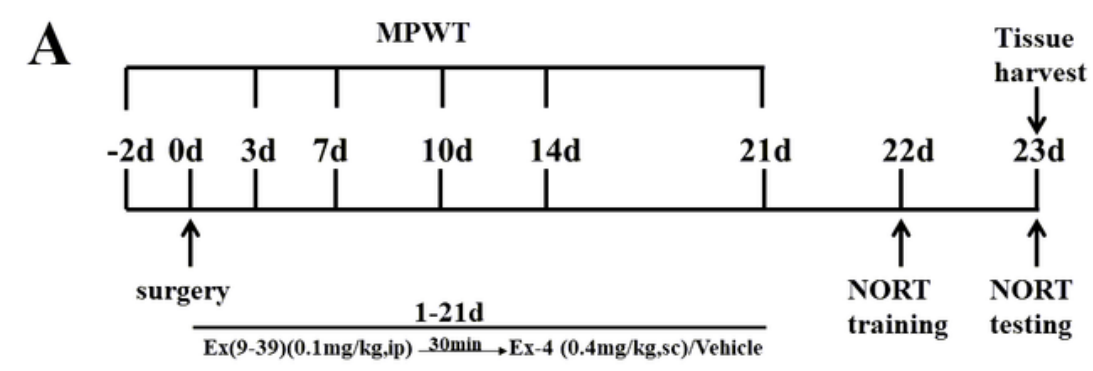

Groups:(1)SNI+Vehicle

(2)SNI+Ex(9-39)

(3)SNI+Ex-4+Vehicle

(4)SNI+Ex-4+Ex(9-39)

\section{B}

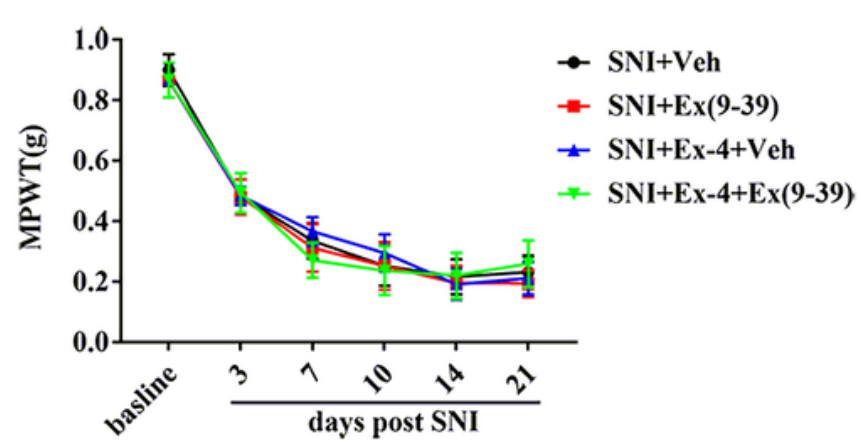

D

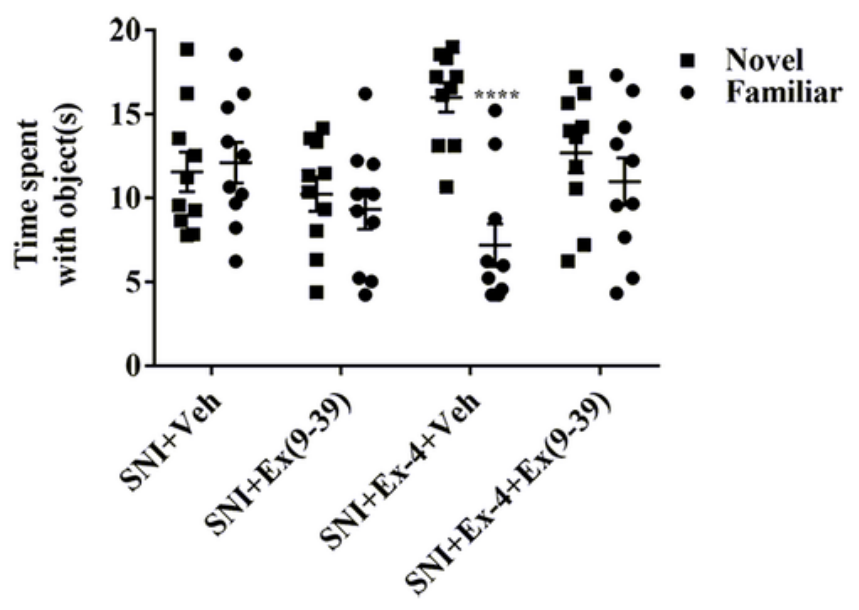

C

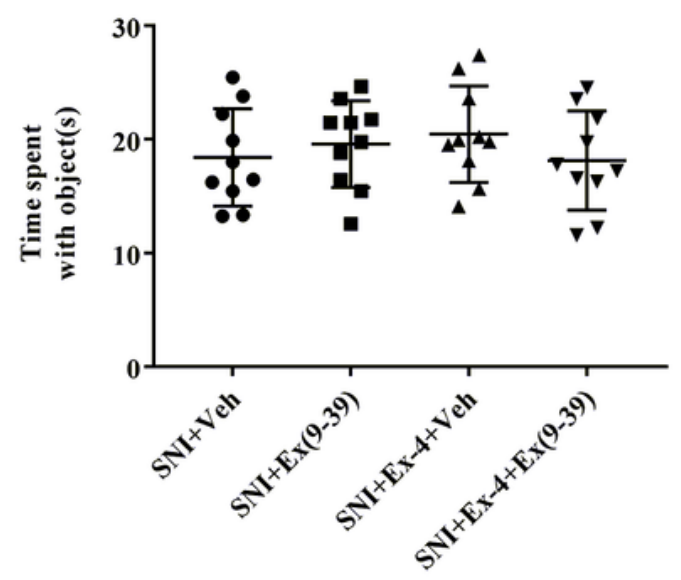

$\mathbf{E}$

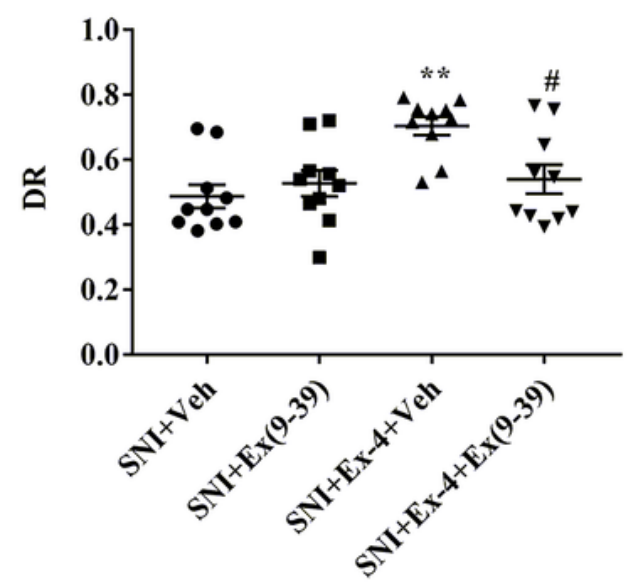

Figure 5

Ex(9-39), a GLP-1R antagonist, reversed the improved recognition memory caused by Ex-4 treatment. a The protocol of application with Ex(9-39) or Ex-4,pain behavior test and NORT. Ex(9-39) was 
treated,30min before Ex-4 administration, from day 1 to 21 after surgery. b Compared with the SNI+Veh group, Ex-4 or Ex(9-39) or Ex-(9-39)+Ex-4 treatment did not change the decreased MPWT induced by $\mathrm{SNI}(\mathrm{n}=12$ mice per group). $\mathrm{c}$ In the NORT training stage, there were no significant differences in the time spent on exploring two identical objects (familiar object) between the four groups( $n=10$ mice per group). $d$ In the NORT testing phase, no significant difference was found in the time for exploring the novel and familiar object, except the SNI+Ex-4+Veh group( $n=10$ mice per group). e Ex-4 could improve the decreased $D R$, but $\operatorname{Ex}(9-39)$ reversed the effects $(n=10$ mice per group). Data are presented as mean \pm $S E M,{ }^{* *} P<0.01,{ }^{* \star * *} P<0.0001, \# p<0.05$. 


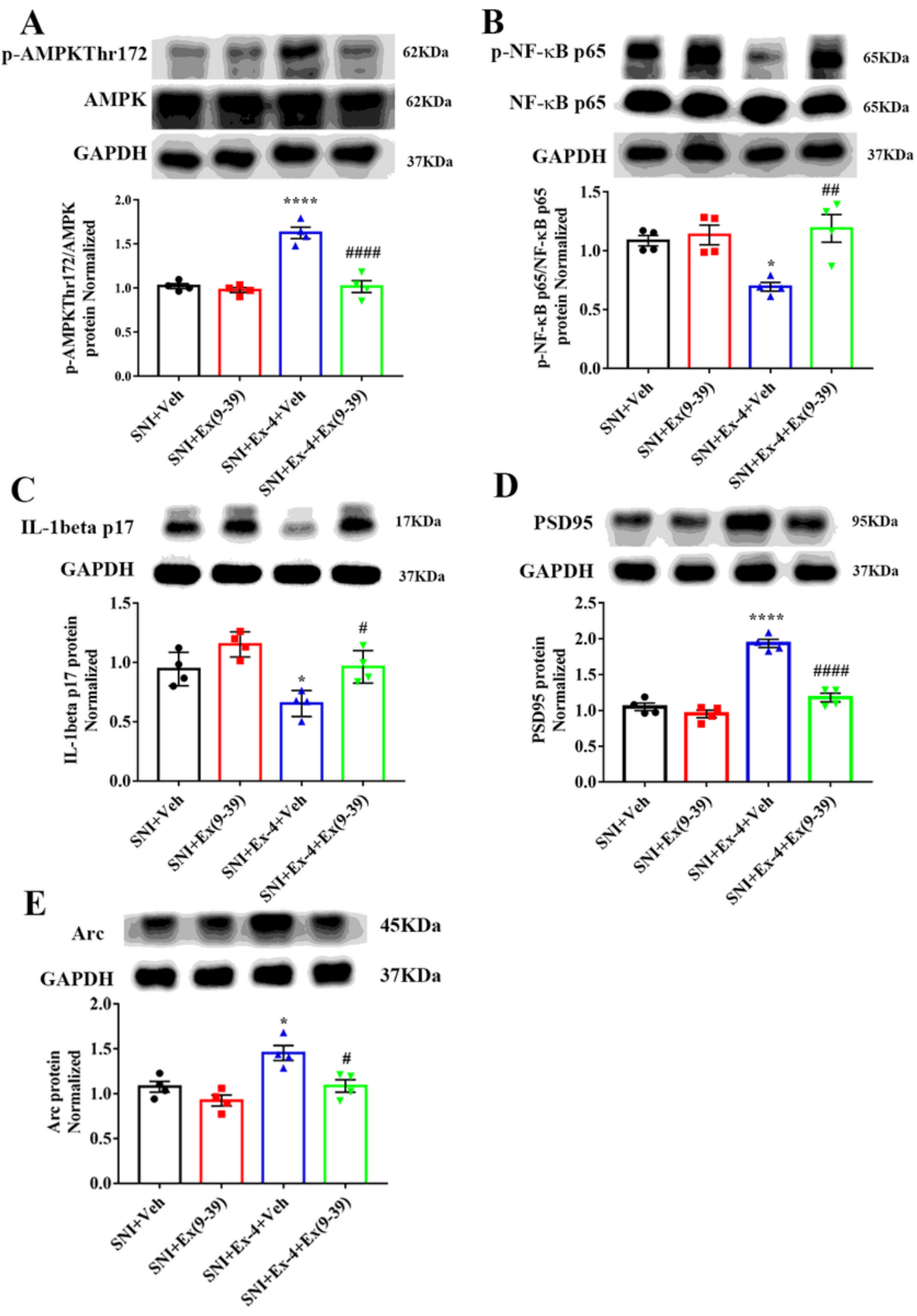

Figure 6

The neuroprotection of Ex-4 was abrogated by treatment with GLP-1R antagonist. a-b Ex(9-39) reversed the attenuated disturbed AMPK/NF-KB signaling compared with SNI+Ex-4+Veh group. $c$ Ex-4 could attenuate neuroinflammation, however; the effect was inhibited with Ex(9-39) application before Ex-4. d-e Ex(9-39) treatment could abolish the upregulated synaptic associated proteins induced by Ex-4 in the 
hippocampus. $n=4$ mice per group. Data are presented as mean $\pm S E M$, ${ }^{*} P<0.05,{ }^{* \star * *} P<0.0001, \# P<0.05, \# \# P<0.01$, \#\#\#\#P<0.0001.
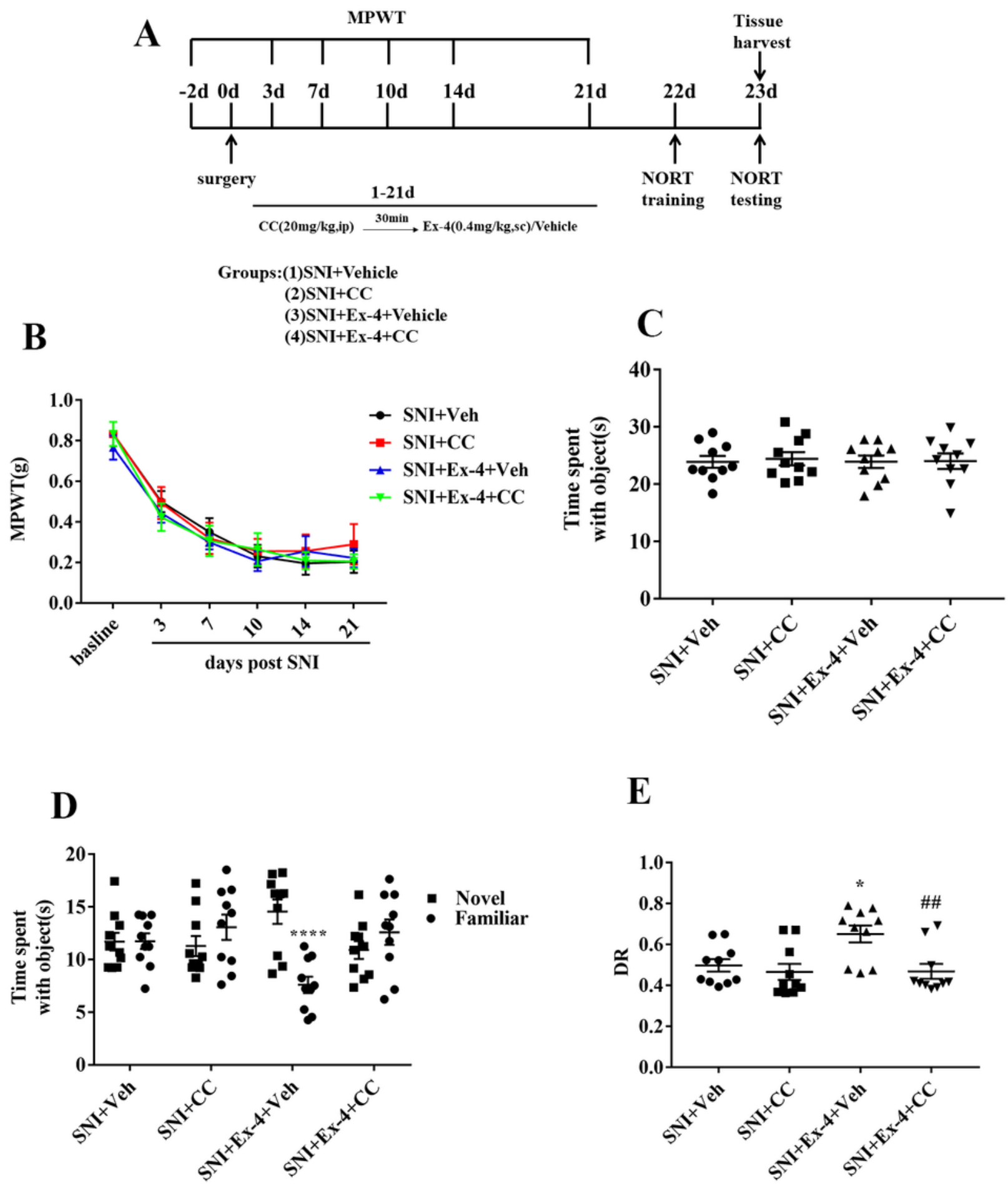

Figure 7

Activation of GLP-1R improved recognition memory dysfunction by activating AMPK. a The schedule of administrating CC or Ex-4,MPWT and NORT. CC, an AMPK inhibitor, was administrated before Ex-4 in SNI mice. b Compared to the SNI+Veh mice,CC,Ex-4 or CC+Ex-4 did not affect the allodynia induced by 
$\mathrm{SNI}(\mathrm{n}=12$ mice per group). $\mathrm{c}$ In the NORT training phase, there were no significant differences in the time spent on exploring two identical objects (familiar object) between the four groups( $n=10$ mice per group). $\mathrm{d}$ In the NORT testing section, a significant difference was found in the time exploring novel objects and familiar objects in the $S \mathrm{NI}+\mathrm{Ex}-4+\mathrm{Veh}$ group compared with the $\mathrm{SNI}+\mathrm{CC}+\mathrm{Ex}-4$ group(n=10 mice per group). e CC+Ex-4 could reverse the increased DR induced by Ex-4 in SNI mice $(n=10$ mice per group).Data are presented as mean $\pm S E M, * P<0.05,{ }^{* \star * *} P<0.0001$, \#\#P<0.01.

A
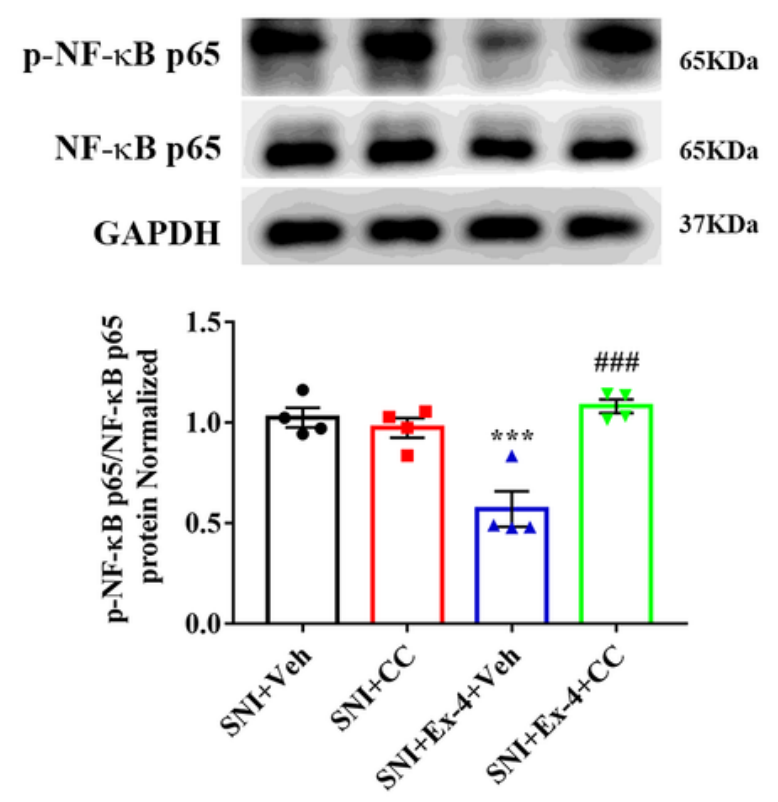

C
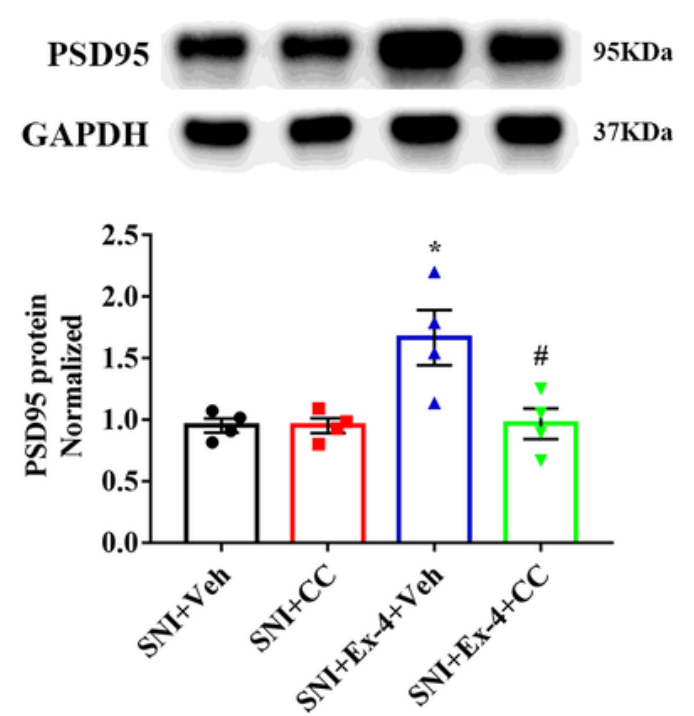

B
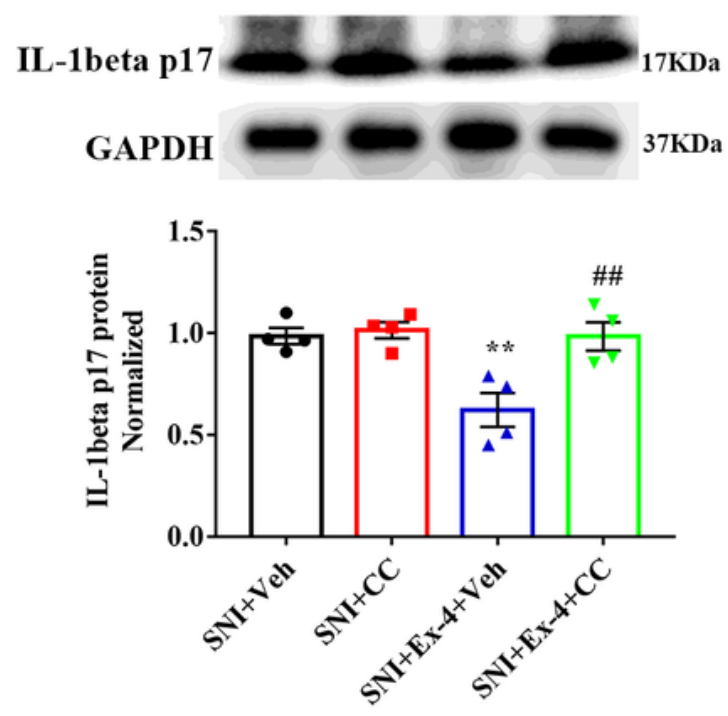

D
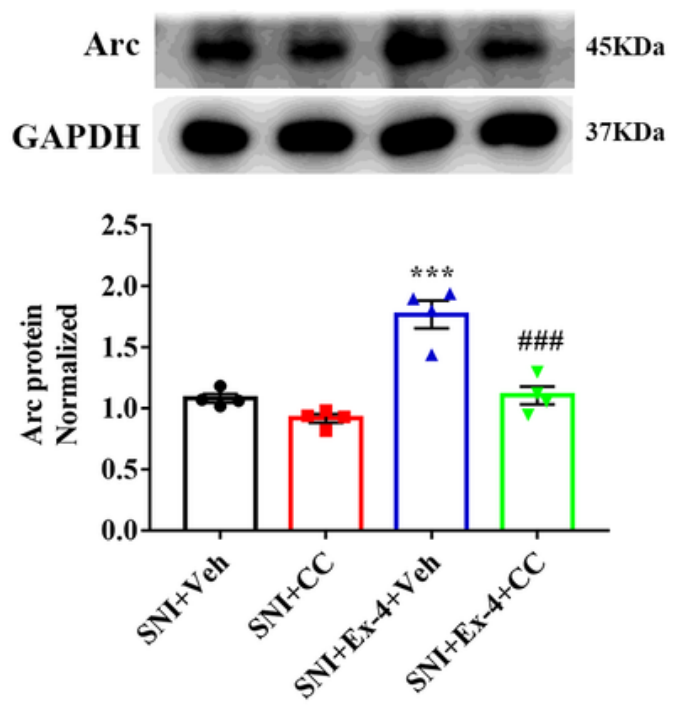

Figure 8 
CC abolished the improved neuroinflammation and synaptic proteins induced by activation of GLP-1R. a Activation of GLP-1R by Ex-4 could downregulate the expression of p-NF-KB p65, but this was reversed by an AMPK inhibitor. $b$ The expression level of IL-1 $1 \beta$ p17 was decreased by activating GLP-1R in the hippocampus; however, the effect was annihilated by administrating CC. c-d Activating GLP-1R could ameliorate the diminished expression levels of PSD95 and Arc in mice memory deficits caused by neuropathic pain, which were annulled by treatment with $\mathrm{CC}(\mathrm{n}=4$ mice per group). Data are presented as mean $\pm S E M,{ }^{\star} P<0.05,{ }^{\star \star} P<0.01,{ }^{\star \star *} P<0.001, \# P<0.05, \# \# P<0.01, \# \# \# P<0.001$ 